\title{
LÍMITES DEL PODER DISCIPLINARIO DEL EMPLEADOR. PRINCIPIOS NON BIS IN IDEM Y DE PROPORCIONALIDAD EN LA IMPOSICIÓN DE SANCIONES LABORALES (SENTENCIA DEL PRIMER JUZGADO DE LETRAS DEL TRABAJO DE SANTIAGO, 8 DE MAYO DE 2013, RIT O- 349- 2013. "MIGUEL ÁNGEL ROJAS REYES CON COMERCIAL MARMICOC S.A.”)
}

\author{
RAÚl FernÁNDEZ TOLEDO*
}

Abogado

\section{EL PODER DISCIPLINARIO DEL EMPLEADOR Y SUS LÍMITES. GENERALIDADES}

La relación jurídica que une al empleador con el trabajador, no obstante tener la naturaleza jurídica de contrato, posee características particulares que no existen en ningún otro contrato ni vínculo jurídico. La misma tiene notas peculiares y distintivas. Ello se debe, principalmente, tanto a la circunstancia que el trabajador presta servicios personales permanentes bajo subordinación del empleador como al interés de este porque los servicios se ejecuten normalmente, procurando que se utilicen correctamente los medios de producción que pone a disposición del trabajador y, asimismo, se mantenga el debido respeto y cordialidad, tanto entre los distintos trabajadores como entre él mismo y sus dependientes.

Con el objeto que el empleador mantenga el orden de los trabajadores al interior de la organización y corrija las conductas contrarias a la disciplina laboral se le reconoce por el ordenamiento jurídico el poder disciplinario ${ }^{1}$. De esta forma, con el poder disciplinario el derecho del trabajo busca otorgar facultades efectivas al empleador, que sean "idóneas para garantizar el desarrollo regular y la continuidad de la actividad de la empresa" ${ }^{2}$, siendo el apoyo indispensable para garantizar el poder de dirección del empleador. Esto porque, si no existiera la posibilidad que el empleador pudiera sancionar conductas de los trabajadores que infringen las instrucciones que imparte, el poder de dirección sería una mera ilusión, al no poder el empresario castigar su transgresión o tener lo posibilidad de ello ${ }^{3}$. El poder disciplinario asegura y garantiza la efectividad del ejercicio del poder de dirección, al permitir al empleador asegurar jurídicamente su autoridad y el cumplimiento de sus órdenes y mandatos, con el objeto de alcanzar el interés de la empresa.

\footnotetext{
* Abogado, 2011. Licenciado en Ciencias Jurídicas y Sociales por la Universidad de Concepción, 2008. Magíster en Derecho del Trabajo y Seguridad Social por la Universidad de Talca y la Universidad de Valencia, 2013. Asociado al Área de Derecho Laboral y Seguridad Social de Philippi Irarrázaval Pulido \& Brunner Abogados. Correo electrónico: raulfernandeztoledo@gmail.com

Lizama Portal, Luis. Derecho del Trabajo. Santiago, Chile: Editorial Lexis Nexis, 2003, p. 80.

Persiani, Mattia; Proia, Giampiero. Diritto del Lavoro. Padua, Italia: Cedam, 2008, p. 242.

Muñiz Fernández-Costales, Javier. Poder disciplinario empresarial y proceso especial de impugnación de sanciones. Oviedo, España: Consejo Económico y Social del Principado de Asturias, 2005, 39.
} 
Raúl Fernández Toledo /Limites del Poder Disciplinario del Empleador.

Principios Non Bis in Idem y de Proporcionalidad en la Imposición de Sanciones Laborales

Muchas han sido las definiciones que se han dado del poder disciplinario, siendo todas, eso sí, coincidentes en su contenido esencial. Se le define como "la facultad que ostenta el empresario para imponer sanciones a los trabajadores como consecuencia de la realización por parte de éstos de conductas que impliquen un incumplimiento de la relación y la disciplina laboral"'. Dicha facultad de imponer sanciones en forma unilateral constituye una excepción al principio de reserva a favor de los poderes públicos del ejercicio del poder sancionador, en el sentido, de que, salvo este poder a favor del empresario, ninguna otra persona o entidad, excepto los poderes públicos, pueden sancionar por incumplimientos contractuales sin acudir, previamente, a los Tribunales de Justicia, sanciones que, además, deberán ser cumplidas por los trabajadores, sin perjuicio de que quepa su posterior control judicial. El mantenimiento y garantía del orden en las relaciones laborales así lo requiere, no siendo conveniente imponerlas a instancias de un órgano externo a la empresa, por cuando el incumplimiento laboral de un trabajador requiere de una respuesta inmediata a la infracción, lo que no ocurriría de esperarse trámites de terceros, que sin duda se dilatarían en el tiempo, especialmente si el órgano es estatal.

La doctrina nacional mayoritaria fundamenta la potestad disciplinaria en el artículo 19 $\mathrm{N}^{\circ} 21$ de la Constitución Política, esto es, en la garantía constitucional de desarrollar cualquier actividad económica ${ }^{2}$. Lo que resulta acertado, puesto que si la libertad económica supone la posibilidad de constitución de empresas y de organización de la actividad empresarial, que permite al empleador organizar los bienes y servicios de la empresa, necesita para ello del poder de dirección. Y este quedaría sin sentido si no tuviera el apoyo del poder disciplinario, los que se configuran como necesarios e indispensables para el correcto funcionamiento de la empresa, sin los cuales sería incongruente hablar de libertad económica. Algunos autores la fundamentan también en el derecho de propiedad reconocido en el artículo $19 \mathrm{~N}^{\circ} 24$ de la Constitución Política ${ }^{3}$. Lo que también se comparte, debido a que el hecho que el empleador arriesgue sus bienes en el desarrollo de una actividad económica en que necesita de trabajadores, hace indispensable que le asistan los poderes de dirección y disciplinario, para evitar perjuicios a su propiedad o evitar que se vuelvan a cometer conductas atentatorias contra la misma.

No te trata de un poder absoluto. Se encuentra sometido a una serie de límites, que tienen como fin proteger al trabajador sancionado en virtud de dicho poder, siendo el más importante el respeto de los derechos fundamentales de los trabajadores, cuando el mismo se pone en movimiento. Punto discutido en la doctrina comparada ${ }^{4}$, y no nacional, donde el poder disciplinario del empleador ha sido escasamente tratado, es la aplicación al poder disciplinario empresarial de los principios que rigen el derecho sancionador estatal. Problema que igualmente existe en el ordenamiento jurídico nacional, por no existir una norma que permita dicha aplicación. A este estado de confusión ha contribuido el hecho que no exista una norma legal ni reglamentaria que establezca claramente cuáles son los límites y/o requisitos del poder disciplinario del empleador. En el Código del Trabajo nacional solamente existen, a nuestro entender, tres normas claras que se refieren a los límites del poder disciplinario. La primera es la contenida en el artículo 5 inciso $1^{\circ}$ del Código del Trabajo, que establece como límite de las facultades del empleador el respeto

\footnotetext{
Poquet Catalá, Raquel. La actual configuración del Poder disciplinario empresarial. Valencia, España: Tirant lo Blanch, 2011, p. 17.

Véase Gamonal Contreras, Sergio; Guidi Moggia, Caterina. Manual del Contrato de Trabajo. Tercera Edición revisada y actualizada. Santiago, Chile: Editorial Legal Publishing, 2012, p. 101.

Lizama Portal, Luis, op. cit. (n. 1), p. 80.

Véase Poquet Catalá, Raquel, op. cit. (n. 4), pp. 42-43.
} 
a los derechos fundamentales del trabajador. La segunda, el artículo $154 \mathrm{~N}^{\circ} 10$, que señala cuáles son los sanciones que puede establecer en el Reglamento Interno el empleador para castigar las infracciones a las obligaciones y prohibiciones que establezca en el mismo. Y finalmente, el artículo 160 que regula las causales de despido que puede invocar el empleador para poner término al contrato de trabajo cuando el trabajador ha incurrido culpablemente en algunas de las conductas tipificadas en el mismo precepto, es decir, cuando ha ejecutado una acción u omisión contraria a la disciplina laboral.

Sin embargo, a pesar de la ausencia de normas que consagren expresamente los límites del poder disciplinario empresarial, la jurisprudencia judicial no ha titubeado en aplicar algunos principios del derecho sancionador estatal, siendo los más frecuentemente aplicados los principios de tipicidad, de proporcionalidad y el non bis in idem, con ciertos matices, debido a la naturaleza propia de la relación laboral, que es de carácter privado. Decisión que resulta acertada. Ello porque el poder disciplinario, como poder que es, supone la aplicación de sanciones por parte del empleador al trabajador infractor de la disciplina laboral, constituyendo una expresión más de la desigualdad jerárquica existente entre las partes de la relación laboral. De ahí, que resulta conveniente sostener que los principios que rigen la potestad sancionadora estatal deben proyectarse sobre el poder disciplinario del empleador. Por lo demás, dichos principios son de carácter general a toda potestad sancionadora, siendo inherentes a la misma, debiendo observarse aun cuando no exista una norma expresa que los consagre.

Atendido el objeto de nuestro comentario de jurisprudencia, debemos detenernos en los principios non bis idem y de proporcionalidad, de amplia aplicación en el derecho penal y administrativo sancionador. El principio non bis in idem conlleva la prohibición de sancionar un mismo hecho respecto de un mismo sujeto y en base a un mismo fundamento más de una vez En virtud de dicho principio se trata de evitar, por un lado, la duplicidad de sanciones sobre unos mismos hechos y, por otro lado, impedir que existan varias sanciones que sancionen doblemente una misma infracción. Por su parte, el principio de proporcionalidad conlleva la obligación de ponderar la gravedad de la conducta del trabajador y los bienes jurídicos afectados, debiendo existir una debida proporción entre la sanción a aplicar y la conducta a sancionar ${ }^{6}$.

A través del presente comentario a la sentencia definitiva dictada por el Primer Juzgado de Letras del Trabajo de Santiago el 8 de mayo de 2013, se pondrá de manifiesto cómo la jurisprudencia judicial ha aplicado los principios non bis idem y de proporcionalidad en los casos en que se ha discutido si el ejercicio del poder disciplinario por parte del empleador fue ajustado a derecho. Y si resulta correcta su aplicación al ámbito disciplinario empresarial.

\section{BREVE RELACIÓN DE LOS HECHOS}

Existió un contrato de trabajo entre don Miguel Ángel Rojas Reyes, en calidad de trabajador, y la empresa Comercial Marmicoc S.A., como empleadora, desde el 06 de junio de 2003 hasta el 05 de diciembre de 2012. La función que cumplía el trabajador era la de encargado de bodega, desempeñándose en la bodega número 3 de la compañía, ubicada en la comuna de Pudahuel, galpón número 3, calle Miraflores $\mathrm{N}^{\circ} 9.699$.

\footnotetext{
Rodríguez-Piñeiro, Manuel. "Potestad Sancionadora y Non Bis in Ídem”, en: Revista Relaciones Laborales, n 20, Madrid, España, 1990, p. 51.

Muñiz Fernández-Costales, Javier, op. cit. (n. 3), p. 57; Gamonal Contreras, Sergio; Guidi Moggia, Caterina, op. cit. (n. 2), p. 103.
} 
Raúl Fernández Toledo /Limites del Poder Disciplinario del Empleador.

Principios Non Bis in Idem y de Proporcionalidad en la Imposición de Sanciones Laborales

Con fecha 5 de diciembre del año 2012, la empleadora puso término al contrato de trabajo de don Miguel Ángel Rojas Reyes en virtud de la causal establecida en el artículo $160 \mathrm{~N}^{\circ} 1$ letra c) del Código del Trabajo, esto es, "Vías de hecho ejercidas por el trabajador en contra del empleador o de cualquier trabajador que se desempeñe en la misma empresa". Los hechos que fundaron el despido del trabajador consistieron en una riña ocurrida al interior de las bodegas de la compañía, en la cual tuvo participación el trabajador junto con otros dos más de la empresa, aproximadamente a las 13:00 horas del día 29 de noviembre de 2012, dentro del horario de trabajo y en donde como resultado de la gravedad de los hechos, se provocaron lesiones a un compañero de la empresa. Las lesiones las sufrió don Eugenio Rojas.

Previo al despido, el día 30 de noviembre de 2012, el señor Miguel Ángel Rojas Reyes fue amonestado por escrito, firmando el documento de la amonestación, en virtud de los mismos hechos que sirvieron de fundamento a su despido.

Como consecuencia del despido, el ex trabajador interpuso demanda de despido injustificado en contra de su ex empleador, solicitando entre otras prestaciones, el pago de la indemnización sustitutiva de aviso previo, indemnización por años de servicios con un recargo del ochenta por ciento.

\subsection{Argumentos PARA ACOGER LA DEMANDA DE DESPIDO INJUSTIFICADO}

El punto central de la discusión se radicó en determinar si el despido del trabajador fue o no justificado. El Primer Juzgado de Letras del Trabajo de Santiago por sentencia definitiva de 8 de mayo de 2012 resolvió que el despido del actor era injustificado, ordenando a su ex-empleadora al pago de las indemnizaciones propias del despido injustificado. Los argumentos esgrimidos por la sentencia para arribar a tal conclusión son los siguientes:

a) Se acreditó que el ex trabajador no incurrió en los hechos imputados en la carta de despido. Si bien en la riña ocurrida en bodegas de la empresa el día 29 de noviembre de 2013, don Eugenio Rojas sufrió lesiones, las mismas no fueron atribuibles al actuar del trabajador despedido, toda vez que se estableció en el juicio que don Miguel Ángel Rojas no propinó ningún golpe a don Eugenio Rojas, sino que fue un tercer sujeto, don Víctor Becerra, quien golpeó al señor Rojas. Por lo demás, el trabajador despedido no provocó a don Eugenio Rojas, sino fue éste último quien inició la agresión. También se estableció que el día 30 de noviembre de 2012 la empleadora amonestó por escrito al trabajador en base a los mismos hechos que justificaron su despido.

b) El tribunal junto con establecer que los hechos imputados en la carta de despido no configuran la causal de caducidad del artículo $160 \mathrm{~N}^{\circ} 1$ letra c) del Código del Trabajo, señala en el considerando décimo de la sentencia como argumento para acoger la demandante, que si la empresa empleadora ya había amonestado, y por ende, sancionado al trabajador por el mismo hecho que justificó su despido, no podía invocar los mismos hechos como fundamento para aplicar una segunda sanción, como lo es el despido del trabajador, ya que se había agotado su poder disciplinario. La empresa al haber ya ejercido su poder disciplinario al amonestar por escrito, no puede, en virtud del principio non bis idem sancionar nuevamente un hecho ya castigado.

c) Agrega la sentencia, que al ser el despido la sanción más grave que contempla el ordenamiento laboral, solamente queda reservado para las faltas que tengan el mismo carácter. De ahí, que de acuerdo al principio de gradualidad, una falta menor amerita la aplicación de 
una sanción leve, en tanto que una falta grave faculta al empleador para aplicar sanciones más onerosas. En el caso de autos se aplicó una sanción grave a una falta leve, vulnerándose un principio que informa el derecho laboral, cual es el principio protector.

\section{COMENTARIO A LA SENTENCIA DEL PRIMER JUZGADO DE LETRAS DEL TRABAJO DE SANTIAGO}

\subsection{PRINCIPIO NON BIS IN IDEM NO OBSERVADO POR EL EMPLEADOR}

Uno de los fundamentos de la sentencia definitiva para declarar injustificado el despido del ex trabajador, fue que el empleador ya había sancionado al trabajador mediante amonestación escrita por los mismos hechos que lo despidió. Es decir, sancionó al trabajador dos veces en base a los mismos hechos, vulnerando el principio no bis in idem, que es uno de los principios que debe observar al castigar a un trabajador.

La invocación del principio non bis in idem como fundamento para declarar injustificado el despido y dar lugar a las indemnizaciones previstas en el artículo 168 del Código del Trabajo, no constituye una cuestión aislada. La jurisprudencia judicial en forma reiterada ha dado aplicación al principio non bis in idem en materia de potestad disciplinaria del empleador. Criterio que se ha mantenido en las últimas sentencias judiciales en donde se ha establecido que el empleador ha sancionado dos veces al trabajador por una misma falta laboral ${ }^{7}$. Eso sí, los fundamentos para su aplicación han sido diversos. En ocasiones, sin señalar más fundamento que la circunstancia, que si bien ha sido desarrollado en materia penal, de acuerdo a la jurisprudencia y doctrina, es igualmente aplicable al ámbito sancionatorio laboral ${ }^{8}$. En cambio, en otras oportunidades, fundándose en el debido proceso, ha agregado:

"sea cual fuere la causal de despido que aplique el empleador para exonerar a un dependiente, si éste es previamente amonestado, la amonestación supone la condonación de la falta, esto es, la renuncia del empleador al derecho de invocarla como fundamento de una extinción, ya que la amonestación es en sí misma una sanción, lo que excluye la aplicación de una segunda, el despido, ya que de hacerlo, se violaría la garantía constitucional del debido proceso, en su manifestación del principio del non bis in idem en materia laboral"9.

\footnotetext{
Sentencia de la Corte de Apelaciones de Santiago, recurso de nulidad, Rol Reforma Laboral N ${ }^{\circ} 1070$ - 2012, 6 de noviembre de 2012, publicada en http://laboral.poderjudicial.cl/SITLAPORWEB/InicioAplicacionPortal.do [consultada el 20 de febrero de 2013]; sentencia de la Corte de Apelaciones de Santiago, recurso de nulidad, Rol Reforma Laboral N $1644-2012,27$ de diciembre de 2012, publicada en http://laboral.poderjudicial.cl/SITLAPORWEB/InicioAplicacionPortal.do [consultada el 20 de febrero de 2013].

8 Sentencia del Segundo Juzgado de Letras del Trabajo de Santiago, juicio despido injustificado, RIT O - 231 - 2009, 26 de noviembre de 2009, publicada en http://www.legalpublishing.cl (Identificador LegalPublising CL/JUR/9331/2009; 44712); sentencia del Segundo Juzgado de Letras del Trabajo de Santiago, juicio despido injustificado, RIT O - 563 - 2009, 8 de enero de 2010, publicada en http://www.legalpublishing.cl (Identificador LegalPublising CL/JUR/14252/2010; 44488).

$9 \quad$ Sentencia del Segundo Juzgado de Letras del Trabajo de Santiago, juicio despido injustificado, RIT O - 468 - 2009, 11 de enero de 2010, publicada en http://www.microjuris.cl/Search (identificador microjuris MJJ 23900); sentencia del Segundo Juzgado de Letras del Trabajo de Santiago, juicio despido injustificado, RIT O - 1064 - 2010, 26 de julio de 2010, publicada en http://www. legalpublishing.cl (Identificador LegalPublising CL/JUR/15220/2010; 46415).
} 
Raúl Fernández Toledo /Limites del Poder Disciplinario del Empleador.

Principios Non Bis in Idem y de Proporcionalidad en la Imposición de Sanciones Laborales

Según se puede observar, la sentencia definitiva del Primer Juzgado de Letras del Trabajo de Santiago, siguiendo un criterio asentado en la jurisprudencia, no señala ninguna norma legal para justificar la aplicación del principio non bis in idem, fundándose en principios generales valiosos para el ordenamiento jurídico. Decisión que resulta acertada. Porque aun cuando en principio, pudiese parecer que es único y exclusivo del ámbito punitivo estatal, es aplicable también a las relaciones laborales, principalmente, al poder disciplinario empresarial. El fundamento de ello, sin perjuicio de lo antes señalado, reside en que se trata de un principio general del derecho, siendo aplicable en todos los ámbitos en los que se desarrolle un poder sancionador, sea por un órgano público o por un sujeto de derecho privado al que el Estado le ha reconocido facultades específicas para aplicar sanciones, tal como ocurre con el empleador en el contexto de las relaciones laborales ${ }^{10}$.

El principio non bis in idem, en relación con el poder disciplinario del empleador, supone reconocer a favor del interés contractual del empresario un sistema de sanciones que surge aparejado al sistema sancionador estatal, lo que implica un juicio de desvalor de la conducta del trabajador, alejándose así de los principios y reglas que inspiran al derecho común de los contratos ${ }^{11}$. Por lo demás, la vigencia del principio nos bis in idem como límite a la potestad disciplinaria empresarial es coherente con la justicia, que se sostiene es uno de los valores superiores del ordenamiento jurídico, y es contrario a dicho valor sancionar dos veces una misma infracción, siendo desproporcionado tal actuar del empleador.

Debe advertirse que la aplicación del principio nos bis idem en materia laboral tiene una serie de matices, no pudiendo aplicarse de igual manera que en sede penal y administrativa. Es así, que debe diferenciarse de la reincidencia infraccional, que no supone una pluralidad punitiva, sino un agravamiento de la conducta del trabajador que previamente ha sido sancionado y, a pesar de esto incide en una nueva conducta infractora que se califica y se sanciona más gravemente ${ }^{12}$. En efecto, el uso de la facultad disciplinaria del empleador, que implica la imposición de sanciones no extintivas del contrato de trabajo, no lo inhabilita para poner término al contrato de trabajo, invocando alguna de las causales de caducidad del artículo $160 \mathrm{~N}^{\circ} 7$ del Código del Trabajo, si el trabajador insiste en mantener la conducta infractora en el tiempo. Así se ha resuelto, tratándose de los atrasos reiterados del trabajador en el cumplimiento de su jornada de trabajo ${ }^{13}$. Tampoco debe confundirse con los casos de infracciones encadenadas, esto es, incumplimientos que se producen de manera consecutiva y que se relacionan con los mismos hechos, si bien las infracciones que se producen son diferenciadas porque también lo son los hechos a los que responden las sanciones a aplicar pueden ser diferentes. En estos casos no se desconoce el principio nos bis in idem, pues se trata de incumplimientos contractuales distintos que son susceptibles de sanciones diferentes ${ }^{14}$.

\footnotetext{
10 Fernández López, María Fernanda. El poder disciplinario en la Empresa. Madrid, España: Editorial Civitas, 1991, pp. 292 y 293; Luque Parra, Manuel. Los límites jurídicos de los poderes empresariales en la relación laboral. Barcelona, España: J.M. Bosch Editor, 1999, p. 488.

11 Rodríguez Rodríguez, Emma. El Poder disciplinario y la Negociación Colectiva. Granada, España: Editorial Comares, 2008, p. 218.

12 Castro Arguelles, María Antonia. El régimen disciplinario en la Empresa. Infracciones y sanciones laborales. Pamplona, Espańa: Aranzadi, 1993, p. 191.

13 Sentencia de la Excma. Corte Suprema, recurso de casación en el fondo, 28 de abril de 2010, Rol No 883 - 2010, publicada en http://www.microjuris.cl/Search (identificador microjuris MJJ23541); sentencia de la Excma. Corte Suprema, recurso de casación en el fondo, 19 de agosto de 2010, Rol N 3729 - 2010, publicada en http://www.microjuris.cl/Search (identificador microjuris MJJ25264).

14 Fernández López, María Fernanda, op. cit. (n. 13), p. 304.
} 
Finalmente, es perfectamente posible compatibilizar las sanciones laborales con la responsabilidad civil del trabajador en supuestos de infracciones laborales culpables o dolosos que causan daño al empleador, esto debido a que la sanción disciplinaria no elimina la responsabilidad civil, sino que ambas deben coordinarse, porque, aunque todas responden al fundamento último del interés empresarial, la disciplinaria tiene como fin castigar, mientras que la civil es resarcir los daños producidos con dicho incumplimiento. De esta manera, al tener cada una un fin diferente, no se conculcaría el principio non bis in idem ${ }^{15}$.

En conclusión, se defiende la vigencia del principio non bis in idem como límite del poder sancionador del empleador, por las razones expuestas. Es por ello, que no obstante, ser escasa la justificación legal de la procedencia de dicho principio en materia sancionatoria empresarial, se estima acertada la decisión de considerar injustificado el despido del trabajador fundado en el principio nos bis in idem. Sin perjuicio de lo señalado, debe reconocerse, eso sí, que es discutible nuestra conclusión y la de la sentencia comentada, debido a que el grueso de la regulación normativa que existe sobre el principio non bis in idem se aplica exclusivamente al ordenamiento sancionador estatal, ya que hace referencia a las infracciones penales o administrativas, es decir, a sanciones impuestas en base al poder punitivo público y no a un poder sancionador privado, como lo es el del empleador.

\subsection{LA PROPORCIONALIDAD EN LA APLICACIÓN DE LA SANCIÓN}

Otro argumento que invoca la sentencia definitiva comentada, está constituido por la circunstancia que no existe proporcionalidad entre la falta cometida por el trabajador y la sanción aplicada. Ello porque, en su concepto, debe existir concordancia entre la falta cometida y la sanción laboral aplicada. De lo contrario se infringe el principio protector.

Si bien resulta ser cierto que constituye un límite del poder disciplinario del empleador la necesaria proporcionalidad entre la falta laboral y la sanción a aplicar por el empleador, yerra la sentencia en la denominación de dicho principio. Puesto que no constituye una expresión del principio protector sino del principio de proporcionalidad. El cual impone la exigencia de que exista una correlación entre la conducta infractora, la sanción y el trabajador sancionado para evitar el uso arbitrario de esta facultad de parte de la empresa ${ }^{16}$.

Es obligatorio el examen individualizado en cada caso concreto donde han de ponderarse todos los elementos concurrentes en él, tanto subjetivos como objetivos: intención del infractor, circunstancias concurrentes, perjuicio al empleador, entre otros. De manera que sólo cuando la conducta del trabajador valorada, teniendo en cuenta todos los elementos de juicio dichos, constituye una infracción de la máxima gravedad resultando procedente el despido, que es también la sanción más grave establecida en el ámbito laboral. De este modo, el empresario sólo puede determinar la sanción concreta a imponer dentro del elenco correspondiente de acuerdo a la gravedad de la infracción concreta de las tipificadas, eliminando así toda posible arbitrariedad y cerrando la puerta a la absoluta decisión unilateral del empleador al momento de imponer la sanción.

Tres son los requisitos o elementos que configuran este principio ${ }^{17}$. En primer lugar, la medida sancionadora debe ser idónea, debiendo resultar apta para castigar al trabajador infractor

\footnotetext{
15 Cremades Sanz-Pastor, Bernardo. La sanción disciplinaria en la Empresa. Madrid, España: Instituto de Estudios Políticos, 1963, p. 115

16 Muñiz Fernández-Costales, Javier, op. cit. (n. 3), p. 57.

17 Terradillos Ormaetxea, Edurne. El Poder disciplinario empresarial. Principios y garantías. Valencia, España: Tirant lo Blanch, 2004, p. 41 y ss.
} 
Raúl Fernández Toledo /Limites del Poder Disciplinario del Empleador.

Principios Non Bis in Idem y de Proporcionalidad en la Imposición de Sanciones Laborales

y evitar la reiteración de la falta sancionada. En segundo lugar, se debe respetar el principio de intervención mínima, esto es, debe valorarse la necesidad de aplicar la sanción laboral, puesto que el orden punitivo se reserva para los ataques más graves e intolerables. Por último, se debe examinar si la sanción en cuestión es ponderada o equilibrada, por derivarse de ellas más beneficios o ventajas para el interés empresarial que perjuicios sobre otros bienes o valores en conflictos.

$\mathrm{Al}$ igual que el principio non bis in idem, no tiene consagración legal de carácter general como límite de la potestad disciplinaria del empleador. Solamente se encuentra implícito en las causales de caducidad previstas en los números 1 y 7 del artículo 160 del Código del Trabajo, que emplean la expresión "grave". Expresión legal que implica, que las faltas laborales no graves pueden no ser sancionadas o bien pueden serlo con sanciones menores no extintivas del contrato de trabajo, como lo son la amonestación verbal, la amonestación escrita o bien multa del veinticinco por ciento de la remuneración diaria del trabajador, de existir reglamento interno en la empresa. La jurisprudencia judicial ${ }^{18}$, tratándose de la causal de incumplimiento grave de las obligaciones que impone el contrato, ha resuelto que no basta que el trabajador incurra en incumplimientos contractuales, sino que es necesario, además, que ellos sean considerados como graves por el tribunal. Agrega, que para ello, el sentenciador de fondo debe efectuar un juicio de proporcionalidad, conjugando diferentes variables que le permitan arribar a la convicción de que, por la entidad de las infracciones contractuales en que ha incurrido el dependiente, resulta de todo mérito sacrificar el vínculo contractual al nivel de su extinción. En otras palabras, para que exista justa causa de despido, en estos casos, el juicio de proporcionalidad debe descartar todas las otras sanciones más benévolas que pudiere aplicar el empleador en ejercicio de sus facultades disciplinarias, y que deben consignarse en el respectivo reglamento interno. Sin embargo, fuera de los preceptos legales señalados, no existen otros. Ante lo cual, su aplicación a la potestad sancionadora del empleador reside en su consideración de principio general del derecho sancionador, lo que supone que debe considerarse como uno de los límites jurídicos que limitan, también, el ejercicio del poder disciplinario empresarial ${ }^{19}$.

Nuestros Tribunales de Justicia en reiteradas ocasiones han aplicación el principio de proporcionalidad en juicios de despido injustificado, declarándolo injustificado cuando resulta vulnerado $^{20}$. Ello porque el mismo obliga a entender que debe existir una cierta proporcionalidad entre el quebrantamiento del deber impuesto al trabajador, y la drasticidad de la sanción aplicada. De no concurrir la proporcionalidad entre la falta y el despido, este debe declararse injustificado

\section{CONCLUSIONES}

En nuestro concepto, el empleador al imponer sanciones a sus trabajadores dependientes debe observar los principios non bis in idem y de proporcionalidad. Sea que imponga una amo-

\footnotetext{
18 En este sentido sentencia de la Corte de Apelaciones de La Serena, recurso de nulidad, Rol Reforma Laboral N 54 - 2013, 1 de julio de 2013, publicada en http://laboral.poderjudicial.cl/SITLAPORWEB/InicioAplicacionPortal.do [consultada el $30 \mathrm{de}$ julio de 2013].

19 Luque Parra, Manuel, op. cit. (n. 13), p. 468.

20 Sentencia de la Corte de Apelaciones de Antofagasta, recurso de apelación, Rol N $242-2005,16$ de enero de 2006, publicada en http://corte.poderjudicial.cl/SITCORTEPORWEB/ (consulta el 10 de junio de 2013); sentencia del Primer Juzgado de Letras del Trabajo de Santiago, juicio despido injustificado, RIT O - 284 - 2010, 5 de mayo de 2010, publicada en http://www.legalpublishing. cl (Identificador LegalPublising CL/JUR/16167/2010; 43837); sentencia del Primer Juzgado de Letras del Trabajo de Santiago, juicio tutela laboral, RIT T - 50 - 2010, 25 de junio de 2010, publicada en http://www.legalpublishing.cl (Identificador LegalPublising CL/JUR/16167/2010; 43838).
} 
nestación verbal, una amonestación escrita, multa del veinticinco por ciento de la remuneración diaria del trabajador sancionado o lo despida por algunas de las causales del artículo 160 del Código del Trabajo, debe necesariamente observar los principios señalados. Si los mismos no son observados, tratándose del despido, necesariamente debe declararse injustificado, por vulnerarse principios inherentes al poder disciplinario empresarial, cuya sanción precisamente es la injustificación del despido. Por su parte, si el empleador aplica una sanción no extintiva del contrato de trabajo y el trabajador sancionado reclama de la misma ante el Juzgado de Letras del Trabajo competente, de acuerdo al artículo 420 letra a) del Código del Trabajo, y se determina que se vulneraron los principios non bis in idem o de proporcionalidad, o ambos a la vez, el juez debe dejar sin efecto la sanción.

Si bien el empleador es quien detenta la facultad exclusiva de elección entre las distintas sanciones previstas por el ordenamiento jurídico, por supuesto dentro del margen establecido por las normas legales, reglamentarias y contractuales que rigen el régimen de faltas y sanciones, como también por los principios que rigen la potestad sancionadora, la eficacia de la sanción queda supeditada a la fiscalización judicial que se haga de ella. Es por ello que resulta acertada la sentencia definitiva comentada al declarar injustificado el despido del trabajador demandante por no observar el empleador los principios nos bis in idem y de proporcionalidad.

\section{BIBLIOGRAFÍA}

Castro Arguelles, María Antonia. El régimen disciplinario en la Empresa. Infracciones y sanciones laborales. Pamplona, España: Aranzadi, 1993.

Cremades Sanz-Pastor, Bernardo. La sanción disciplinaria en la Empresa. Madrid, España: Instituto de Estudios Políticos, 1963.

Fernández López, María Fernanda. El poder disciplinario en la Empresa. Madrid, España: Editorial Civitas, 1991.

Gamonal Contreras, Sergio; Guidi Moggia, Caterina. Manual del Contrato de Trabajo. Tercera Edición revisada y actualizada. Santiago, Chile: Editorial Legal Publishing, 2012.

Lizama Portal, Luis. Derecho del Trabajo. Santiago, Chile: Editorial Lexis Nexis, 2003.

Luque Parra, Manuel. Los límites jurídicos de los poderes empresariales en la relación laboral. Barcelona, España: J.M. Bosch Editor, 1999.

Muñiz Fernández-Costales, Javier. Poder disciplinario empresarial y proceso especial de impugnación de sanciones. Oviedo, Espańa: Consejo Económico y Social del Principado de Asturias, 2005.

Persiani, Mattia; Proia, Giampiero. Diritto del Lavoro. Padua, Italia: Cedam, 2008.

Poquet Catalá, Raquel. La actual configuración del Poder disciplinario empresarial. Valencia, España: Tirant lo Blanch, 2011.

Rodríguez Rodríguez, Emma. El Poder disciplinario y la Negociación Colectiva. Granada, España: Editorial Comares, 2008.

Rodríguez-Piñeiro, Manuel. “Potestad Sancionadora y Non Bis in Ídem”, en: Revista Relaciones Laborales, n 20, Madrid, España, 1990.

Terradillos Ormaetxea, Edurne. El Poder disciplinario empresarial. Principios y garantías. Valencia, España: Tirant lo Blanch, 2004. 